\title{
Leadless Pacemakers: practice and promise in congenital heart disease
}

\author{
T.S.O. Clarke ${ }^{1}$, A.M. Zaidi ${ }^{2}$ and B. Clarke $2,3,4^{*}$
}

\begin{abstract}
The development of the implantable cardiac pacemaker nearly 60 years ago represented a paradigm shift in the control of potentially lethal heart rhythms and has revolutionized the lives of those at risk of sudden death from bradycardia. Subsequent technological developments have resulted in devices that offer additional benefits to patients, including defibrillation and resychronisation therapy. Previously, Cardiac Implantable Electronic Devices (CIEDs) relied on leads, either transvenous or epicardial, to transmit electronic impulses to the heart. The potential value of self-contained leadless intracardiac pacemaker devices has long be recognised with their potential to avoid intrinsic problems with traditional CIEDs, particularly device-related infection and lead failure. Two leadless devices have been introduced since 2012 which may offer a safe, long term alternative to leaded CIEDs with greater patient acceptability and greater flexibility, particularly for congenital heart disease patients where venous access problems may otherwise limit access to traditional transvenous systems. Here we examine the development of leadless pacemakers, the current state of affairs in clinical practice, and key considerations for future developments, with a focus on the usage of such devices in the setting of congenital heart disease.
\end{abstract}

Keywords: Pacemaker, Leadless, Development, Congenital, Review, Cardiac implantable electronic device

\section{Background}

Although the vast majority of patients requiring CIED implantation have structurally normal hearts, paediatric and adult patients with congenital cardiac abnormalities represent one of the most challenging groups for deviceimplanting cardiologists. Congenital heart disease patients commonly have abnormalities of the conduction system (e.g. congenital atrioventricular block occurs in $25 \%$ of patients with congenitally corrected transposition of the great arteries), either directly related to developmental abnormalities or as a result of surgical correction [1]. High risk of cardiac sudden death associated with congenital abnormalities is also recognized as an indication for ICD implantation in the 2014 NICE guidelines. Persistent left-sided superior vena cava (SVC) may be present in $4.3-11 \%$ of patients with congenital heart disease compared with $0.3-0.5 \%$ of the general population [2]. In over $90 \%$ of cases, the left-sided SVC runs

\footnotetext{
* Correspondence: Bernard.clarke@manchester.ac.uk

${ }^{2}$ Manchester Heart Centre, Manchester Royal Infirmary, University of

Manchester, Manchester, United Kingdom

${ }^{3}$ Royal Manchester Children's Hospital and Academic Health Science Centre,

University of Manchester, Manchester, United Kingdom

Full list of author information is available at the end of the article
}

into the coronary sinus and drains into the right atrium but may drain into the left atrium. The majority (90\%) of patients will have bilateral SVC. Delivery of pacing leads via the CS is often technically difficult but feasible although pacing via a bridging communication to the right SVC (present in around 30\% of cases) is often more straightforward. If left-sided implantation is not possible, then $90 \%$ of patients have a right SVC. Even if the SVC approach is feasible, constraints imposed by anatomical abnormalities or surgical correction may make conventional pacemaker implantation impossible (e.g. tricuspid atresia or Fontan repair) but in many patients pacing is technically possible but may carry higher short-term and long-term risks than in the general population [3]. In children, there is the additional problem of growth of the child which means either placing an excessively large loop on the pacing leads to accommodate growth which may predispose to arrhythmias or accepting the need to revise systems during childhood [4].

Femoral (trans-inferior vena cava) pacing may be an option in some patients were access via the SVC is not feasible $[5,6]$ and epicardial pacing is widely used in ACHD patients but unless corrective surgery is also 
being undertaken, requires additional surgery and necessitates sternotomy if atrial pacing is required. Longevity of epicardial leads is also a concern $[7,8]$.

The development of fully implantable pacemakers in the late 1950s was one of the key developments in modern cardiology and remains the only proven treatment for symptomatic bradycardia [9]. There are around 700,000 pacemaker implants each year worldwide [10]. Technological advances have resulted in reduced device size allowing implantation in the precordium rather than the abdomen and allowed multi-chamber, programmable devices lasting 7-10 years with sophisticated functionality allowing more physiological pacing. However, the basic design for a pacemaker system has not changed since its introduction into clinical practice. The electrical pulse is supplied by a pulse generator containing the electronics and battery implanted surgically into a subcutaneous pocket in the precordium, connected to one or more insulated lead(s) inserted via the subclavian vein and superior vena cava to the endocardial surface of the chamber to be paced. Conventional pacemaker treatment continues to be associated with complications. Immediate post-operative adverse events occur in at least $10 \%$ of patients and are either related to the surgical procedure to implant the pulse generator (haematoma, skin erosion, pocket infection) or result from venous access and transvenous lead placement (pneumothorax, cardiac perforation, lead dislodgement) [11]. Long-term complications are also common, with studies suggesting a 5-year complication rate up to $20 \%$, mainly due to lead problems $(11 \%)$ and pocket problems (8\%) [12]. The pacing lead remains the most vulnerable component of the system, with risks of fracture and insulation failure as well as connector issues. Younger, more active patients place more stresses on pacing leads and this may be a particular issue in children and younger ACHD patients. The steadily increasing numbers of implants and the longer life expectancy of ACHD patients will inevitably increase long-term complications.

The idea of a pacemaker that could be implanted directly into the heart, avoiding the need for creation of a pocket and an unsightly surgical scar and eliminating the pacemaker lead has long been considered the ideal solution by physicians. The first totally self-contained leadless pacemaker system was proposed by Spickler in 1970 [13], using a device powered by mercury-zinc and nuclear power with pacing electrodes being directly placed on the pacemaker capsule and implanted with a catheter-based system inserted through the femoral vein. It was successfully tested in animals but limitations in battery technology and electronics prevented progression of the system to human trials. It has taken over four decades to achieve the necessary $>90 \%$ size reduction of the integrated pacing device without compromising the functionality expected in modern pacemakers (i.e. satisfactory longevity, access to MR scanning, rate adaptation and external communication).

The first leadless pacemaker (Nanostim LP, St. Jude Medical, Sylmar, CA, USA) became available in 2012. It is an entirely self-contained, active-fixation, rate-adaptive pacemaker, $42 \mathrm{~mm}$ in length with a maximum diameter of $5.99 \mathrm{~mm}$ (18 F) (Fig. 1). The device is delivered to the right ventricle at the end of a purpose-designed steerable percutaneous delivery catheter via the femoral vein. Initially, it was recommended that it was placed at the right ventricular apex but complications in the first tranche of implants lead to revision of the protocol advising implant on the apical septum $[14,15]$. The Nanostim is anchored with the use of a helical screw-in fixation electrode at the distal end of the device with a secondary fixation mechanism using nylon tines. The on-going LEADLESS II trial [16], a prospective, nonrandomized, multicenter study, recently reported the primary analysis of efficacy and safety in the initial 300 patients receiving the Nanostim who were followed for 6 months and outcomes for all 526 patients who were enrolled as of June 2015. Implant success was $95.7 \%$. Device-related serious adverse events were observed in $6.7 \%$ of the 300 patients in the primary cohort, including device dislodgement in $1.7 \%$, cardiac perforation in $1.3 \%$, elevated pacing thresholds requiring device retrieval and reimplantation in 1.3\%, and vascular complications in $1.3 \%$. The authors compared the complications of Nanostim implantation favourably with implantation of conventional ventricular pacemakers where total complications are reported to be

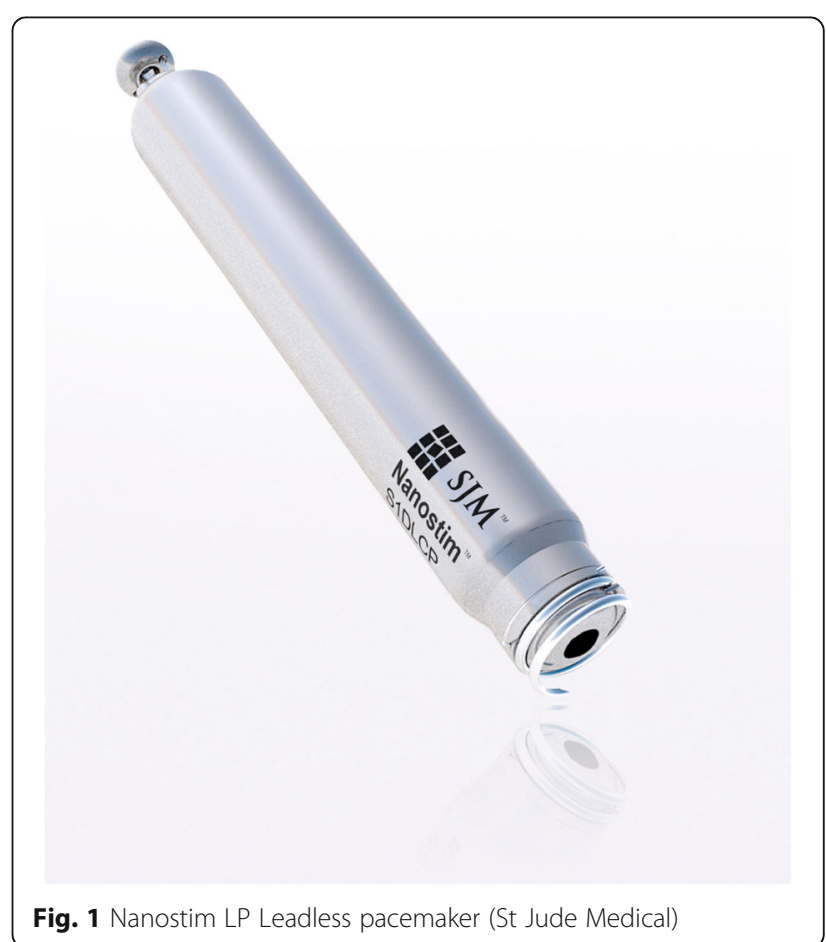


$3.2 \%$ including pneumothorax in $1.1 \%$, lead displacement in $0.8 \%$ and infection in $0.5 \%$ [17]. The main concern with Nanostim is cardiac perforation leading to tamponade. Early reports suggested a perforation rate up to $4 \%$ leading to revision of the implant protocol but overall the perforation rate was $1.5 \%$ in the 526 patients in the total cohort. This appears comparable to the rate observed with transvenous leads $(0.6$ to $5.0 \%)[11,18,19]$. but the large diameter of the Nanostim is more likely to cause clinically significant tamponade and is a particular concern in the older patient group which represents the largest group of potential recipients for the current single chamber device.

The other currently-available leadless pacing system is the Micra ${ }^{\mathrm{TM}}$ transcatheter pacing system (TPS, Model MC1VR01, Medtronic plc, Mounds View, MN, USA) (Fig. 2). It is a $0.8 \mathrm{~cm}^{3}, 2.0 \mathrm{~g}$, self-contained, hermetically enclosed capsule, single-chamber ventricular pacemaker with functionality and features similar to existing ventricular pacemakers, inclusive of rate responsive pacing and automated pacing capture threshold management. The device is $25.9 \mathrm{~mm}$ in length, with an outer diameter of $6.7 \mathrm{~mm}$. It is inserted using a steerable transfemoral catheter delivery system through a 23 French internal diameter/27 French outer diameter introducer. The device is fixed into the endocardium using four electrically inactive protractible nitinol tines located on the distal end of the device. The absence of a screw for fixation reduces the risk of perforation but may be more problematic in implant sites without significant cardiac trabeculation. This may be a limiting factor in some ACHD patients although the device is actively rather

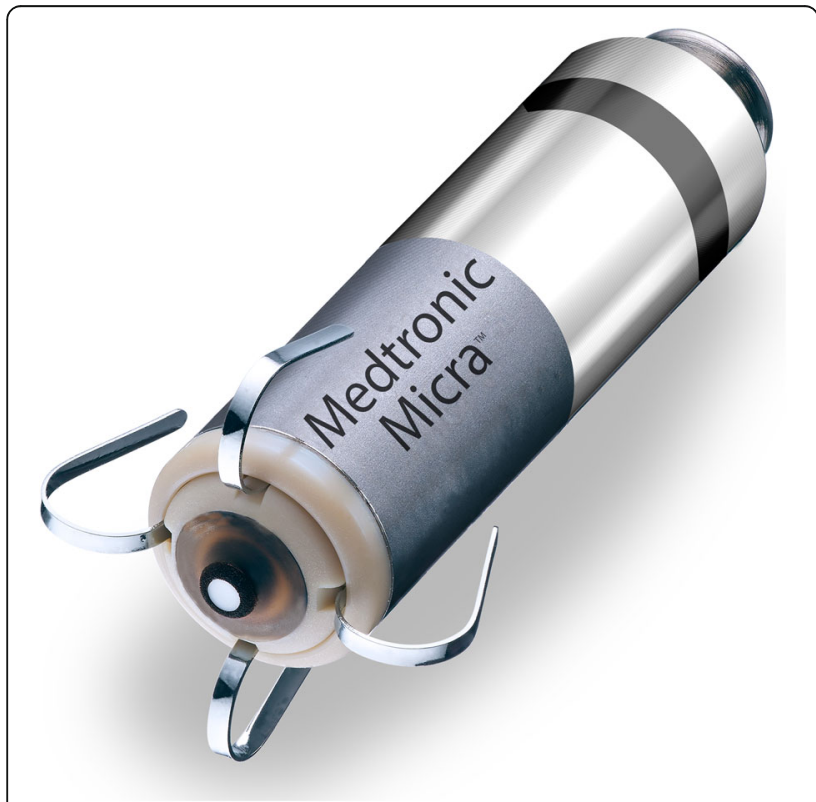

Fig. 2 Medtronic Micra transcatheter pacing system (TPS) than passively fixed despite the absence of a helix and has been successfully implanted on the high septum without complication, something that is not normally possible with passive tined pacing leads. The recent report of Micra performance showed 100\% implant success, but an $18.6 \%$ rate of procedure-related adverse events. Perforation was less of a problem than with Nanostim (0.7 vs $1.5 \%$ ) but the rate of vascular complications was higher, including bleeding in $2.1 \%$ of patients, haematoma in $1.4 \%$, and pseudoaneurysm in $1.4 \%$ [20]. This reflects the larger caliber of the implant sheath $(27 \mathrm{~F})$ for the shorter, wider Micra than the $16 \mathrm{~F}$ sheath used for the longer, thinner Nanostim. The Nanostim is noticeably more mobile within the right ventricular cavity but this does not appear to be associated with clinically significant arrhythmias. Compared with Micra, the Nanostim device is of similar size but longer $(41.4 \mathrm{v} 25.9 \mathrm{~mm})$ and narrower in diameter (18 vs $20 \mathrm{~F})$. Rate response is controlled by RV blood temperature compared with an accelerometer in the Micra. At present, the Micra is interrogated using the programmer head and the standard Medtronic 2090 programmer. The Medtronic Carelink remote monitoring system can not be used with the Micra at present, but will be shortly. The Nanostim uses a novel conductive communication system using surface ECG electrodes to wirelessly communicate with the St. Jude Medical programmer and will be available on the Merlin ${ }^{\mathrm{TM}}$ remote monitoring system shortly.

The limited duration of follow-up with leadless devices makes it difficult to accurately predict battery longevity but battery depletion for leadless devices should be slower than for conventional devices due to the absence of leads and developments in low-power electronics and battery technology which are likely to offset the smaller size of leadless device batteries. The estimated average longevity of the Nanostim is 15.0 years [16] and the Micra 12.6 years [20] which exceeds the lifespan of conventional devices. The most difficult question that remains to be definitively answered regarding leadless devices is what to do when the battery is depleted. Ideally, the device should be safely retrievable at end of service but endothelialisation will inevitably occur and extraction of conventional pacing leads in situ for more than 8 years is proven to be associated with increased risks of cardiac injury. Simply implanting another device next to the depleted device may be reasonable in the ventricle (though probably not in the atrium) because of the small size of the leadless devices. Leadless devices are easily manually programmed off and automatically deactivate at end of service. There are limited data regarding retrievability. Conventional active fixation pacemaker leads are known to be safer to extract than tined leads and one would therefore expect the screw-in Nanostim to be readily extractable. Similarly, the flexible 
nitinol tines on Micra are designed to allow extraction by simple traction. In the Leadless II trial, the Nanostim was successfully retrieved in seven patients up to 413 days post-implant without complications [16]. Data are more limited for Micra. A study in four sheep implanted with a Micra prototype reported that all four devices were successfully retrieved and had remained relatively free from encapsulation at their proximal ends. Histological analyses demonstrated only minimal damage to cardiac tissues [21].

It is highly probable that further developments in leadless technology will lead to even smaller devices in the future and that abandoning depleted devices will become more widely accepted. The ability to safely abandon leadless devices in the heart may ultimately be an advantage over leaded systems. Pulse generator changes and lead revisions have been repeatedly been shown to be associated with particularly high risks of device infection and younger patients who will inevitably require multiple pacemaker procedures in their lifetime will consequently be predisposed to multiple extraction procedures for lead failure or infection. In the REPLACE Registry, major complications occurred in $15.3 \%$ of patients undergoing planned transvenous lead addition for replacement or upgrade but were higher in patients who had an upgrade to or a revised cardiac resynchronization therapy device (18.7\%) [22]. Indeed, one of the most encouraging aspects of initial experience with leadless pacemakers has been the absence of infections in the studies.

Leadless pacemakers have other potential advantages over traditional systems. The lack of an extended antenna (i.e. lead) and limited content of ferromagnetic components makes leadless pacemakers much more readily MR-conditional [23]. Both Nanostim and Micra are safe for full body magnetic resonance imaging in 1.5 and 3.0 T scanners and it is likely that they will be suitable for scanning in more powerful magnets in the future $[16,20]$. They will certainly be more able to keep pace with developments in MR technology than traditional leaded devices. Cardiac MR is firmly established as the most valuable imaging modality in ACHD patients and increased use of leadless pacemakers will have definite advantages in the congenital group. It also offers significant benefits in patients with significant tricuspid regurgitation and particularly patients who have undergone tricuspid annuloplasty. Although passing a large bore catheter through the tricuspid valve may be technically challenging after surgical repair, the ability to pace the right ventricle without having to leave a lead traversing the valve would be advantageous. Trial data shows that implant times are short (mean time of implant $28.6 \pm 18.7 \mathrm{~min}$ for Nanostim [16] and $37 \pm 21 \mathrm{~min}$ for Micra [20]) which compares well with standard leaded VVI implants.
They certainly offer an expectation of better long-term pacing results than epicardial or femoral pacemakers. In a recent study of epicardial pacemakers in patients less than 18 years of age, the probability of absence of any pacing system dysfunction was 70.1 and $47.2 \%$ at 5 and 10 years after implantation, respectively [8]. Similarly, femoral pacing is often complicated by lead instability. In the largest series of femoral pacemakers in 90 patients, atrial lead displacement was common (21\%) despite use of active fixation leads and ventricular lead displacement was also common (7\%) [5].

Cost is the issue most likely to limit their use at present. The initial cost of the leadless pacemaker is £6-7000 (i.e. approximately ten times the cost of a standard leaded VVI). It is very unlikely that an economic case can be made for routine use of leadless devices in the usual VVI population (i.e. elderly patients with atrial fibrillation). However, it is far easier to make the case to use leadless devices in younger ACHD patients where the long-term benefits are likely to be greater and where the cost of epicardial procedures may offset the higher cost of leadless devices.

One major issue is the lack of any relevant trial data concerning the use of leadless pacemakers for paediatric and ACHD patients. All studies involving Nanostim and Micra have required subjects to be over 18 years. ACHD patients have not so far been included and the smallest patient to receive Micra weighed $41 \mathrm{~kg}$. Additionally, both trials had a small number of entrants in comparison to the several hundred thousand lead-based pacemaker procedures performed each year. For congenital patients, who may require clinical management from birth, the ages of participants involved in the LEADLESS II (76 \pm 8 years) and Micra trials $(77.0 \pm 10.2$ years) are very different to the paediatric group that may be best served by a leadless device. The consideration that the differences in anatomy and physiology between the elderly subjects in these trials and a young child with a congenital disorder are significant, so more data are required before any translation of these results into the clinical paediatric or young ACHD population can confidently occur. The long term sequelae of implantation of multiple leadless devices in younger patients, particularly those with complex anatomical or physiological issues remain unknown. The dimensions of the subpulmonary ventricle in Transposition of the Great Arteries may be a particular concern with use of relatively bulky leadless delivery systems and devices.

The major concern about leadless CIED implantation in the paediatric group, however, is femoral sheath size. The current sheath size is $16 \mathrm{~F}$ for the Nanostim and $27 \mathrm{~F}$ for the Micra. There is data suggesting that haemostasis can be achieved in children as young as 3 years old following insertion of sheaths up to $16 \mathrm{~F}$ using a 
subcutaneous "figure of 8" suture [24] but concerns remain about the larger sheath used for Micra.

One of the most exciting possibilities is that leadless pacemakers will be able to overcome the key limitations of the subcutaneous ICD (implantable cardioverterdefibrillator) (SICD, Cameron Health) [25] which has gained widespread acceptance as a useful tool in management of young patients, including congenital patients, at risk of sudden cardiac death. The second generation device is thinner and offers an effective alternative to traditional ICDs especially where venous access or anatomy prevent implantation of transvenous leads. The major weakness of the SICD is that it does not offer bradycardia pacing (although it does allow a short period of post-shock, albeit transcutaneous, pacing) or antitachycardia pacing, leaving high-output shocks as the only treatment it can offer for ventricular tachyarrhtyhmias. The possibility of communication between a leadless pacemaker and SICD raises the possibility of adding bradycardia and anti-tachycardia pacing to the device, greatly increasing its capabilities.

Currently, Nanostim and Micra offer only VVIR pacing. Single-chamber pacing accounts for only $8-25 \%$ of the leaded pacemaker market [2]. Development of multi-chamber leadless systems is essential if the technology is to be widely applicable. There are a number of technological challenges. Fixation in the thinner-walled atrium and coronary sinus must inevitably be different to the ventricle and the sensors in each chamber must be able to communicate effectively without outside interference. One option for a dual-chamber device would be to have a connection between the atrial and ventricular sensors but this would negate the advantages of not having a lead passing through the tricuspid valve. Independent sensors in the different chambers will potentially increase the cost of the pacing system significantly, while abandoning depleted components in the atrium or coronary sinus would potentially be more problematic than in the ventricle. Nonetheless, multichamber devices are likely to appear within the next few years and will offer better functionality for younger, more active patients.

Other leadless devices are in development. Promising results have been reported with an intravascular defibrillator comprising a right ventricular (RV) single-coil lead and titanium electrodes in the superior vena cava-brachiocephalic vein region and the inferior vena cava (InnerPulse, Inc, Research Triangle Park, North Carolina USA) [26] while an ultrasound-based $\mathrm{WiCS}^{\mathrm{TM}}$ system (Wireless Cardiac Stimulation; EBR Systems, Sunnyvale, CA, USA) has been proposed as a means of offering LV pacing which is not reliant on CS anatomy and is not constrained by procedural issues and the long-term risk of thromboembolic events related to the lead permanently residing inside the LV. It comprises a device receiving its energy from a subcutaneous ultrasound transmitter providing LV capture. The WiSE-CRT study [27], a multicenter, prospective study in heart failure patients with an indication for CRT reported successful implantation in 13 out of 17 patients but device-related pericardial effusions occurred in three patients. Nonetheless, effective biventricular pacing was achieved in 11 of the 12 evaluable patients (92\%) at 6 months associated with improvements in LV function and NYHA class suggesting that further developments of the technology may have clinical applications in the future.

\section{Conclusions}

The development of miniaturised leadless pacemakers implanted directly into the heart via the femoral vein represents a new era in device therapy. They have the potential to make pacing simpler and safer, particularly for younger patients and may offer ACHD patients a better solution to their pacing needs than epicardial or femoral pacing. More data are needed about the safety and long-term performance of these devices and questions remain about end-of-service issues but it is likely that leadless technology will be one of the most exciting developments in paediatric and ACHD pacing for decades.

\section{Abbreviations}

ACHD: Adult Congenital Heart Disease; CIEDS: Cardiac implantable electronic devices; CS: Coronary sinus; ICD: Implantable Cardioverter-Defibrillator; LV: Left ventricle; MR: Magnetic Resonance; NICE: National Institute for Health and Care Excellence; NYHA: New York Heart Association Functional Classification; RV: Right ventricle; SICD: Subcutaneous Implantable Cardioverter Defibrillator; SVC: Superior Vena Cava; WI: Ventricular pacing, ventricular sensing: pacing inhibited if beat sensed; WIR: Ventricular pacing, ventricular sensing: pacing inhibited if beat sensed; physiological response to exercise

\section{Acknowledgements}

We thank St Jude Medical and Medtronic Inc for permission to use Figs. 1 and 2.

\section{Funding}

No funding was required for this article.

\section{Availability of data and materials}

Data sharing not applicable to this article as no datasets were generated or analysed during the current study. This is a review article.

\section{Authors' contributions}

TSOC researched and wrote the main draft of the manuscript and both AZ and $\mathrm{BC}$ edited the final versions. All authors read and approved the final manuscript.

\section{Author's information}

Theo SO Clarke (TSOC) BSC (Hons.) is a medical student at Barts and the London School of Medicine and Dentistry. Amir M Zaidi (AMZ) BSC (Hons,) MBChB (Hons.) FRCP is Consultant Cardiologist and Lead for Device therapy at Manchester Heart Centre, Manchester Royal Infirmary. Bernard Clarke BSC MB ChB MD FRCP FESC FACC FRAeS FRCOG (hon.) is Consultant Cardiologist at Manchester Heart Centre, Manchester Royal Infirmary, Royal Manchester Children's Hospital and Honorary Clinical Professor of Cardiology at the University of Manchester Academic Health Science Centre. 


\section{Ethics approval and consent to participate}

Not applicable.

\section{Consent for publication}

Not applicable.

\section{Competing interests}

AZ has received educational support from St Jude Medical, Medtronic and Cook Medical. AZ and BC are Nanostim and Micra implanters. TSOC has no competing interests.

\section{Publisher's Note}

Springer Nature remains neutral with regard to jurisdictional claims in published maps and institutional affiliations.

\section{Author details}

'Barts and the London School of Medicine and Dentistry, Queen Mary University of London, London, United Kingdom. ${ }^{2}$ Manchester Heart Centre, Manchester Royal Infirmary, University of Manchester, Manchester, United Kingdom. ${ }^{3}$ Royal Manchester Children's Hospital and Academic Health Science Centre, University of Manchester, Manchester, United Kingdom. ${ }^{4}$ Central Manchester University Hospitals NHS Foundation Trust, Oxford Road, Manchester, United KingdomM13 9WL.

Received: 6 December 2015 Accepted: 26 June 2017

Published online: 17 July 2017

\section{References}

1. Rutledge JM, Nihill MR, Fraser CD, et al. Outcome of 121 patients with congenitally corrected transposition of the great arteries. Pediatr Cardiol. 2002:23:137-45.

2. Sarodia BD, Stoller JK. Persistent left superior vena cava: case report and literature review. Respir Care. 2000:45:411-16.

3. Smerup $M$, Hjertholm $T$, Johnsen SP, et al. Pacemaker implantation after congenital heart surgery: risk and prognosis in a population-based followup study. Eur J Cardiothorac Surg. 2005;28(1):61-8.

4. Silka MJ, Bar-Cohen Y. Pacemakers and implantable cardioverterdefibrillators in pediatric patients. Heart Rhythm. 2006;3(11):1360-6.

5. Ellestad MH, Caso R, Greenberg PS. Permanent pacemaker implantation using the femoral vein: a preliminary report. Pacing Clin Electrophysiol. 1980;3:418-22.

6. Mathur G, Stables RH, Heaven D, Ingram A, Sutton R. Permanent pacemaker implantation via the femoral vein: an alternative in cases with contraindications to the pectoral approach. Europace. 2001:3:56-9.

7. Kubus P, Materna O, Gebauer RA, Matejka T, Gebauer R, Tlaskal T, Janousek J. Permanent epicardial pacing in children: long-term results and factors modifying outcome. Europace. 2012;14:509-14.

8. Paech C, Kostelka M, Dähnert I, Flosdorff P, Riede FT, Gebauer RA. Performance of steroid eluting bipolar epicardial leads in pediatric and congenital heart disease patients: 15 years of single center experience. J Cardiothorac Surg. 2014;9:84-9.

9. Epstein AE, DiMarco JP, Ellenbogen KA, et al. ACC/AHA/HRS 2008 Guidelines for Device-Based Therapy of Cardiac Rhythm Abnormalities: a report of the American College of Cardiology/American Heart Association Task Force on Practice Guidelines (Writing Committee to Revise the ACC/AHA/NASPE 2002 Guideline Update for Implantation of Cardiac Pacemakers and Antiarrhythmia Devices) developed in collaboration with the American Association for Thoracic Surgery and Society of Thoracic Surgeons. J Am Coll Cardiol. 2008:51(21):e1-e62.

10. Mond HG, Proclemer A. The 11th world survey of cardiac pacing and implantable cardioverter-defibrillators: calendar year 2009 - a World Society of Arrhythmia's project. Pacing Clin Electrophysiol. 2011;34:1013-27.

11. Kirkfeldt RE, Johansen JB, Nohr EA, Jørgensen OD, Nielsen JC. Complications after cardiac implantable electronic device implantations: an analysis of a complete, nationwide cohort in Denmark. Eur Heart J. 2014;35:1186-94.

12. Udo EO, Zuithoff NPA, van Hemel NM, de Cock CC, Hendriks T, Doevendans PA, Moons KG. Incidence and predictors of short- and long-term complications in pacemaker therapy: The FOLLOWPACE study. Heart Rhythm. 2012;9:728-35

13. Spickler JW, Rasor NS, Kezdi P, Misra SN, Robins KE, LeBoeuf C. Totally selfcontained intracardiac pacemaker. J Electrocardiol. 1970;3:325-31.
14. Reddy VY, Knops RE, Sperzel J, Miller MA, Petru J, Simon J, et al Permanent leadless cardiac pacing: results of the LEADLESS trial. Circulation. 2014;129:1466-71.

15. Knops RE, Tjong FVY, Neuzil $P$, et al. Chronic performance of a leadless cardiac pacemaker: 1-year follow-up of the LEADLESS trial. J Am Coll Cardiol. 2015;65:1497-504

16. Reddy W, Exner DV, Cantillon DJ, Doshi R, Bunch TJ, Tomassoni GF, Friedman PA, Estes III NAM, Ip J, Niazi I, Plunkitt K, Banker R, Porterfield J, Ip JE, Dukkipati SR for the LEADLESS II Study Investigators. Percutaneous implantation of an entirely intracardiac leadless pacemaker. N Eng J Med. doi:10.1056/NEJMoa1507192

17. Healey JS, Toff WD, Lamas GA, et al. Cardiovascular outcomes with atrial based pacing compared with ventricular pacing: meta-analysis of randomized trials, using individual patient data. Circulation. 2006;114:11-7.

18. Mahapatra S, Bybee KA, Bunch TJ, et al. Incidence and predictors of cardiac perforation after permanent pacemaker placement. Heart Rhythm. 2005:2:907-11.

19. Acha MR, Keaney JJ, Lubitz SA, et al. Increased perforation risk with an MRI conditional pacing lead: a single-center study. Pacing Clin Electrophysiol. 2015:38:334-42

20. Ritter P, Duray GZ, Steinwender C, et al. Early performance of a miniaturized leadless cardiac pacemaker: the Micra Transcatheter Pacing Study. Eur Heart J. 2015. (Epub ahead of print)

21. Bonner M, Neafus N, Byrd C, Schaerf R, Goode L. Extraction of the Micra transcatheter pacemaker system (abstract). Heart Rhythm. 2014;11:S342.

22. Poole JE, Gleva MJ, Mela T, Chung MK, Uslan DZ, Borge R, Gottipaty V, Shinn T, Dan D, Feldman LA, Seide H, Winston SA, Gallagher JJ, Langberg J, Mitchell K, Holcomb R for the REPLACE Registry Investigators. Complication rates associated with pacemaker or implantable cardioverter-defibrillator generator replacements and upgrade procedures. Results from the replace registry. Circulation. 2010;122:1553-61.

23. Wilkoff BL, Bello D, Taborsky M, et al. Magnetic resonance imaging in patients with a pacemaker system designed for the magnetic resonance environment. Heart Rhythm. 2011;8:65-73.

24. Zhou Y, Guo Z, Bai Y, Zhao X, Qin Y, Chen S, Wu H, Huang X, Li S, Liu B. Femoral venous hemostasis in children using the technique of "figure-ofeight" sutures. Congenit Heart Dis. 2014:9:122-5.

25. Bardy GH, Smith WM, Hood MA, Crozier IG, et al. An entirely subcutaneous implantable cardioverter-defibrillator. N Eng J Med. 2010;363:36-44.

26. Neuzil P, Reddy W, Merkely B, Geller L, Molnar L, Bednarek J, et al. Implantable intravascular defibrillator: defibrillation thresholds of an intravascular cardioverterdefibrillator compared with those of a conventional ICD in humans. Heart Rhythm. 2014;11:210-5.

27. Auricchio A, Delnoy PP, Butter C, Brachmann J, Van Erven L, Setal S. Feasibility, safety, and short-term outcome of leadless ultrasound-based endocardial left ventricular resynchronization in heart failure patients: results of the Wireless Stimulation Endocardially for CRT (WiSE-CRT) study. Europace. 2014;16:681-8

\section{Submit your next manuscript to BioMed Central and we will help you at every step:}

- We accept pre-submission inquiries

- Our selector tool helps you to find the most relevant journal

- We provide round the clock customer support

- Convenient online submission

- Thorough peer review

- Inclusion in PubMed and all major indexing services

- Maximum visibility for your research

Submit your manuscript at www.biomedcentral.com/submit
C) Biomed Central 